\title{
Avatars de la différence sexuelle à la fin de la Renaissance
}

Ian MacLean

\section{(2) OpenEdition}

\section{Journals}

\section{Édition électronique}

URL : http://journals.openedition.org/shakespeare/1194

DOI : 10.4000/shakespeare.1194

ISSN : 2271-6424

Éditeur

Société Française Shakespeare

\section{Édition imprimée}

Date de publication : 1 novembre 1991

Pagination : 113-124

Référence électronique

Ian MacLean, «Avatars de la différence sexuelle à la fin de la Renaissance », Actes des congrès de la Société française Shakespeare [En ligne], 9| 1991, mis en ligne le 01 janvier 2007, consulté le 06 mai 2019. URL : http://journals.openedition.org/shakespeare/1194 ; DOI : 10.4000/shakespeare.1194 


\section{SHAKESPEARE \\ ET LE CORPS A LA RENAISSANCE}

Société Française Shakespeare

Actes du Congrès 1990

sous la direction

de

M.T. JONES-DAVIES

PARIS

LES BELLES LETTRES

1991 
Tous droits de traduction, de reproduction et d'adaptation réservés pour tous les pays.

(C) 1991 Société d'édition Les Belles Lettres, 95 bd Raspail 75006 Paris

ISBN $2.25169117-\mathrm{X}$ 


\section{Avatars de la différence sexuelle à la fin de la Renaissance}

Il n'y a rien de plus osé que de se camper au beau milieu de ce qui est la mode intellectuelle; et rien de plus à la mode aujourd'hui que sexualité, corps, et différence (avec ou sans a): or j'ai entrepris de parler des trois ensemble. On me pardonnera, j'espère, ma témérité. Il va de soi que la différence sexuelle influe puissamment sur les discours théologique, médical, juridique, politique, poétique de 1600; c'est un vaste terrain de recherches que j'ai essayé ailleurs de défricher ${ }^{1}$. Je ne me livrerai pas aujourd'hui à une tâche de cartographe, étant donné le peu de temps dont je dispose; je ne parlerai que de quelques textes philosophiques, médicaux et politiques. Mais en écartant les autres disciplines (et surtout la théologie), je ne cherche pas à nier que toutes les sciences humaines à cette époque s'appuient, s'imbriquent, s'entrepénètrent. Je ne nierai pas non plus que celui dont cette société honore le nom n'entre quaccessoirement dans mes propos, car je veux parler de textes humanistes qui ne peuvent influer que fort indirectement sur celui qui, selon Ben Jonson, ne possédait que "peu de latin et encore moins de grec"2. Je parlerai surtout du contexte aristotélicien; car si (et on l'a souvent dit) Platon est l'âme de la Renaissance, Aristote en est le corps, non seulement parce qu'il s'occupe du monde sublunaire alors que son ancien précepteur porte ses regards vers les cieux, mais aussi parce qu'il fournit aux penseurs de la fin du XVI ${ }^{e}$ siècle une philosophie universelle et cohérente; le fait qu'il y a sur ses ouvrages plus de 1400 commentaires écrits au cours de ce siècle (contre à peu près 10 sur les dialogues de Platon) en témoigne assez clairement ${ }^{3}$. On verra que ses idées sur la différence sexuelle sont contestées; mais il n'en reste pas moins vrai qu'elles constituent en quelque sorte la pierre de touche de la pensée de cette époque. Aristote lui-même expose (avant de les réfuter) les idées de Pythagore et de Platon; ses idées à lui sont mises en doute par Galien; les érudits de la Renaissance cherchent à mettre de l'ordre dans ce domaine fort encombré. Suivons donc les progrès de ce débat. 
Comment penser la différence sexuelle? Il n'y a guère d'époque qui ne se soit penchée sur cette question. Les termes mâle et femelle forment une opposition indispensable à ceux qui cherchent à expliquer l'ordre du monde; c'est du moins le cas de l'ancienne pensée grecque telle qu'Aristote nous l'a transmise. Dans sa Métaphysique il nous décrit le système de l'école de Pythagore, système où s'opposent en deux séries des termes qui se répartissent selon la bipolarité mâle-femelle. Du côté du mâle, donc, les termes ou concepts fini, impair, un, droite, carré, immobile, ligne droite, jour, bien; de l'autre côté, femelle, infini (imparfait), pair, pluralité, gauche, oblong, mobile, courbe, nuit, mal. Qu'on ne cherche pas ici une justification logique ou empirique de ce classement quasiarbitraire, où les propositions mathématiques se juxtaposent à des termes d'ordre naturel ou moral. Ce système décrit par Aristote dans sa Métaphysique n'attire pas de longs commentaires de la part des exégètes du XVIe siècle; les oppositions de Pythagore ont néanmoins laissé leurs traces par le truchement d'Hippocrate. Les écrits de ce dernier, source de connaissances sur la médecine, sont vénérés par les humanistes; on y retrouve liés les termes femelle / pair / gauche, et mâle / impair / droite. Selon ses Aphorismes, c'est la semence du testicule droit qui engendre le mâle, et l'embryon de ce sexe se logera dans le côté droit de l'utérus. La femelle est engendrée et logée de la manière opposée. Selon Hippocrate on peut savoir qu'un enfant sera inéluctablement mort-né s'il vient au monde pendant le huitième mois de la grossesse, car selon le célèbre triangle de Pythagore (dont les côté mesurent 3,4 et 5) huit veut dire un doublement ou du principe mâle et impair $(3+5)$ ou du principe femelle et pair $(4+4)$, combinaisons qui ne peuvent être qu'infertiles. Mais les grossesses qui durent 7 ou 9 mois ont tout en leur faveur, car les principes mâle et femelle y sont réunis $(3+4 ; 5+4)$. On retrouve ces affirmations assez souvent chez les médecins respectés du XVI ${ }^{\mathrm{e}}$ siècle, tel Levinius Lemnius, qui s'en sert dans son célèbre traité sur les merveilles secrètes de la nature $(1559)^{4}$. L'intérêt qu'on montre pour de telles formules est lié au désir presque universel d'engendrer des mâles et d'éviter les fausses couches: il y a naturellement d'autres recettes (ô combien plus saugrenues) pour s'assurer une descendance masculine, qui ne nous concernent pas aujourd'hui, car elles ne relèvent pas des oppositions de Pythagore.

On ne retrouve pas non plus trace de ses idées chez Platon: on verra que ses déclarations sur la différence sexuelle touchent au domaine politique ê moral bien plus qu'à celui de la physique ou 
de la biologie. C'est Aristote lui-même qui va approfondir les recherches sur la physiologie sexuelle en même temps qu'il met de l'ordre dans le concept d'opposition. Nous allons donc écarter le maître, sans pourtant le perdre de vue, pour parler de l'écolier. Chez Aristote, l'étude des principes mâle et femelle est plus fouillée, plus empirique, plus logique; mais on peut encore lire en palimpseste les oppositions de Pythagore. Chez le Stagirite, on remarque une tendance générale à élaborer des dualités hiérarchisées, dont un terme est supérieur à l'autre, ou en quelque sorte le précède : c'est le cas de l'opposition mâle/femelle. Le principe mâle est lié à des principes positifs : activité, forme, perfection; tandis que le principe femelle s'associe à imperfection, passivité, privation, matière : c'est un principe qui appète le mâle pour trouver là sa complétion 5 .

En physiologie aristotélicienne, l'opposition mâle/femelle s'apparente étroitement à la dualité chaud/froid (ou plus exactement plus chaud / plus froid) et à celle d'âme et de corps (principes indifférenciables au niveau hylomorphique, mais séparables au niveau métaphysique et théorique). Aristote finit donc par créer deux séries de termes qui rappellent les séries de Pythagore : mâle/forme/ acte/perfection/possession/chaleur/âme : femelle/matière/puissance /imperfection/privation/froideur/corps. C'est la privation ou l'imperfection chez la femelle qui est la source de son désir de perfection (c'est-à-dire, de son désir de conjonction sexuelle). Mais Aristote admet dans son système un élément de comparaison ou de relation (chaleur/ froideur) qui peut être vérifié empiriquement, et qui agit en tant que structure explicative. Selon lui, l'être le plus parfait est le plus chaud et le plus fini. Un tel être est le mâle de toute espèce biologique : c'est celui qui implante sa semence dans la femelle afin d'engendrer des êtres parfaits de son espèce : c'est-àdire des mâles. La femelle apporte dans ses menstrues, qui peuvent d'ailleurs rester intérieures, la matière dont sera formé l'embryon; la semence du mâle apporte la forme. De plus, c'est dans la semence mâle que réside le principe de l'âme. Il y a certes une semence femelle, mais elle ne sert qu'à apporter à l'embryon la matière. Si la semence femelle prévaut sur la semence mâle, l'enfant ressemblera plus à la mère qu'au père. C'est ainsi qu'Aristote échappe à la conclusion qui serait autrement inéluctable alors qu'elle est niée par l'expérience, que tout enfant ressemblera plus ou moins à son père. Le but de la Nature dans l'acte de génération, c'est de reproduire des êtres parfaits en leur espèce, c'est-à-dire des mâles mais si l'acte même manque de chaleur, ou s'il s'effectue 
sous des conditions peu propices (temps, climat, régime des parents peuvent y jouer un rôle), alors la perfection de l'espèce n'est pas accomplie et une femelle en résulte : c'est-à-dire un être privé de la perfection mâle (íoti rempó́pevov selon Aristote), mais capable de contribuer la matière à la reproduction de l'espèce) et d'être le lieu même où cela s'accomplira ${ }^{6}$. Dans ce système se cachent quelques éléments du système de Pythagore : mâle s'y trouve associé à immobile et fini, femelle à mobile et infini. Pour en trouver des échos plus lointains mais plus puissants, il faut se reporter au livre de l'histoire des animaux d'Aristote où il parle de la psychologie ou attributs mentaux des deux sexes:

Les femelles ont toujours moins de courage que les mâles sauf chez l'ours et la panthère. Chez ces derniers, c'est la femelle qui semble la plus courageuse. Dans les autres genres, les femelles sont plus douces, plus rusées, moins simples, elles ont plus de vivacité et sont plus attentives à élever leurs petits, tandis que les mâles sont plus braves, plus féroces, plus simples et moins rusés.

Ces traits de caractère se remarquent pour ainsi dire chez tous les animaux, mais ils sont plus nets chez ceux dont le caractère est plus complexe, et plus particulièrement chez l'homme. C'est chez ce demier, en effet, que le caractère atteint sa perfection. Aussi estce chez lui que ces diverses manières d'être se distinguent le mieux. C'est pourquoi la femme est plus compatissante que l'homme, plus sujette aux larmes : elle est aussi plus jalouse et plus portée à se plaindre de son sort; elle distribue plus facilement les injures et coups. La femme cède également plus que l'homme au découragement et au désespoir; elle est plus effrontée et plus menteuse; elle est plus facile à tromper et oublie moins vite : elle a en outre moins besoin de sommeil, mais est moins active : d'ailleurs, d'une manière générale, la femelle est moins empressée à agir que le mâle, et il lui faut moins de nourriture ... (Historia animalium, ix.1 [608 a 21 sqq.]).

Tout n'est pas ici au désavantage des femelles, car on voit qu'elles sont censées être plus compatissantes; mais la raison a moins de prise sur elles, d'où il s'ensuit qu'elles sont plus volages, plus légères, plus infidèles, et plus menteuses que les mâles. On retrouve donc ici l'association femelle-mal attribuée par Aristote à Pythagore : association qui s'insinue dans le discours poétique de l'époque de Shakespeare en France comme en Angleterre. Citons à titre d'exemple Maurice de la Porte, auteur d'un livre alphabétique d'épithètes qu'il a soigneusement recueillis chez les poètes de la 
Pléiade, et publiés en 1573. Sous le vocable 'femme' on trouve les termes suivants :

Muable, tromperesse, cheveluë, malitieuse, compagne de l'homme, belle, pusillanime, gente, babillarde, fine vengeresse, sotte, volage, indiscrette, furieuse, superbe, reuesche, mammeluë, enuieuse, passionnee, barguignarde, chiche, desdaigneuse, charmeresse, imbecille, double, fiere, delicate, legere, inutile, venimeuse, lasiue, fardee, ialouse, fascheuse, impatiente, mensongere, propre, desloyale, mesnagere, auare, fragile, mauuaise, obstinee, soupçonneuse, incorrigible, paoureuse, aspre, mariee, quereleuse, vile, dissoluë, perruquee, variable, impudique, fraudulente ou frauduleuse, criarde, assotee, pleureuse ou pleurarde, cauteleuse, passion de l'homme, miserable. ${ }^{7}$

Sous ce torrent d'épithètes arbitrairement juxtaposés, on entrevoit non seulement l'effet de la bipolarité pythagorique mais aussi les conséquences en termes psychologiques de l'opposition aristotélicienne plus chaud/plus froid.

Il faut cependant noter une grande différence entre le système de Pythagore et celui d'Aristote, qui en voulant réfuter les arguties des philosophes mégariens a formulé une taxinomie de la différence. Dans ses ouvrages sur la logique, le Stagirite distingue qutre types d'oppositions ou d'antithèses: d'abord l'opposition de termes qui sont relatifs l'un à l'autre (par exemple, double et moitié, ou toute comparaison corrélative, comme plus chaud/plus froid); deuxièmement l'opposition de la contrariété qui est celle de termes qui font partie d'un même genre mais qui different le plus entre eux (tels noir et blanc); en troisième lieu, l'opposition de la possession et de la privation (par exemple ouie et surdité); enfin l'opposition de la contradiction, fondatrice de toute logique, qui sépare deux propositions dont l'une nie et l'autre affirme ${ }^{8}$. Ce qui nous importe le plus dans l'analyse de la différence sexuelle, ce sont deux types d'oppositions : en premier lieu l'opposition de privation, sous laquelle, on l'a déjà noté, se range la différence sexuelle selon Aristote; en deuxième lieu, l'opposition de termes corrélatifs qui a aussi son rôle à jouer, car l'antithèse plus chaud/plus froid appartient à ce type. Or il y a un risque d'incohérence logique dans la combinaison de privation et de relation; mais il y a aussi un gain, car l'antithèse plus chaud/plus froid peut permettre au philosophe d'incorporer dans son système les observations qui enregistrent la diversité des individus membres de la même espèce. Par exemple, les hommes les plus chauds doivent ex hypothesi être plus chaud que les femmes les plus chaudes . mais il existe des femmes plus 
chaudes que certains hommes, ceux qui sont relativement froids en tant qu'hommes. Ainsi les sexes se chevauchent (si j'ose dire).

Le médecin grec Galien écrivant au II ${ }^{e}$ siècle après J.-C. saisit cette échappatoire et en fait la clé de voûte de son paradigme sexuel. Dans ses ouvrages sur la semence, et sur l'usage des parties du corps, il accepte la différence relative dans la chaleur des sexes, et la rènd tributaire d'un fonctionnalisme général de la Nature. Selon lui, la Nature ne fait rien d'inutile : si la femelle est plus froide que le mâle, on doit écarter l'hypothèse d'une différence de privation qui transforme la femelle en ouvrage imparfait de la Nature, et chercher dans la différence relative une explication naturaliste. La froideur chez la femelle ne peut être que fonctionnelle, en ce qu'elle lui permet de consumer moins vite la nourriture, car la femelle doit à son tour nourrir l'embryon qu'elle porte. La semence des deux sexes contient en puissance et la forme et la matière du foetus; les conditions qui dominent au roment de la conjonction des semences déterminent le sexe de "'epunt et sa ressemblance à l'un ou à l'autre parent. Ce n'est pas is rivation chez la femelle qui la pousse à désirer le mâle: comme Galien l'affirme fort justement d'après ses observations empiriques à ce propos, ce désir est commun aux deux sexes; il est implanté par la Nature dans chaque créature. Bien d'autres "faits" physiologiques s'expliquent par cette froideur transformée en principe fonctionnel : les menstrues, la calvitie chez les mâles (c'est leur chaleur intérieure qui "consume" les cheveux), la voix fluette chez la femelle, sa chair plus dense, plus molle, plus grasse qui fait qu'elle brûle bien mieux que le mâle sur les bûchers funéraires; enfin ses hanches plus amples et sa carrure moins large, qui résultent du manque de force inhérent à la matière plus froide dont elle est formée. Ce manque de vertu propulsive est cause aussi de sa raison plus débile, la matière parvenue à la hauteur de la tête étant insuffisante pour constituer un cerveau aussi puissant que celui du mâle ${ }^{9}$.

Ces idées aristotéliciennes et galéniques sont très discutées au $\mathrm{XVI}^{\mathrm{e}}$-siècle, et les médecins de cette époque élaborent une riche problématique sur la relation entre monstruosité et féminité, l'imperfection des femmes, la semence mâle et femelle, la détermination du sexe chez l'embryon, la spécificité sexuelle quant à la physiologie et à la psychologie. Je n'ai pas le temps ici de passer en revue ces questions, ni d'esquisser les réponses ingénieuses d'un Luis Mercado, d'un Girolamo Mercuriale, d'un André du Laurens : qu'il suffise d'affirmer qu'à la fin du siècle, partout en Europe, Galien est devenu le champion de ceux qui maintiennent que la 
femelle (et à plus forte raison, la femme) n'est pas une version imparfaite du mâle mais son corrélatif dans l'ordre de la génération de l'espèce, aussi parfaite que lui dans son sexe. Mais comme on l'a vu, cette différence relative qui rejette l'idée de la privation et qui établit l'égalité des sexes quant à la physiologie entraîne aussi une infériorité psychologique. Il y a quelques médecins (fort peu, il faut l'admettre) qui proposent l'égalité des sexes quant aux opérations de l'esprit; mais la plupart déduisent de cette froideur relative de la femelle des effets psychologiquess nuisibles à la rationalité. On peut ainsi en arriver à la conclusion que la Nature veut que les femmes soient moins rationnelles que les hommes; d'où il s'ensuit que les rôles politiques et sociaux traditionnellement impartis aux deux sexes ont une justification "naturelle". L'homme, plus chaud, plus robuste, plus constant, plus stable, plus résolu, plus raisonnable, est destiné à jouer un rôle public et actif dans la vie; tandis qu'il sied à la femme, chez qui ces qualités sont moindres, de rester à la maison à garder enfants et domestiques sous la tutelle du mâle ${ }^{10}$.

Nous voilà sortis du discours médical, où règne un système essentiellement péripatéticien : dans le domaine du discours politique et moral où nous ont menés ces débats sur la psychologie, les idées de Platon et des stoïciens entrent en jeu. Selon eux, le corps n'influe sur l'esprit que par l'intermédiaire des passions et de la volonté. Il n'y a point de distinction entre les sexes quant à l'esprit qui est immatériel : il n'y a donc pas de différence psychologique innée. Ces idées platoniques et stoïques sont fort répandues en Europe : on n'a qu'à penser aux noms de Ficin, de Pic de la Mirandole, de Vives, d'Erasme et plus tard de Cardan, de Montaigne, de Juste-Lipse et de Guillaume du Vair. L'idéalisme optimiste incarné dans ce système s'exprime le plus clairement chez Descartes, qui dans son Traité des passions de 1649 déclare qu'il n'y a point d'âme si faible qu'elle ne puisse, étant bien conduite, acquérir un pouvoir absolu sur ses passions"11. On retrouve ici la conséquence logique de la thèse platonique (ou plutôt socratique) de l'identité de vertu chez les hommes et les femmes : et si la vertu est une et même, il doit s'ensuivre que les rôles politiques le sont aussi, ou devraient l'être : thèse contestée explicitement par Aristote, comme on s'y attendrait, dans les termes suivants :

La tempérance n'est pas la même chez la femme et chez l'homme, ni non plus le courage, ni la justice, comme le pensait Socrate; chez l'un le courage est tout de commandement, chez l'autre tout de soumission; et il en est de même des antres vertus ... il faut donc 
croire que les vers du poète [Sophocle] au sujet de la femme contient une vérité générale : "Pour la femme le silence est une parure"... mais non pour lhomme.

$(\text { Politica i. } 5[1260 \text { a } 20 \text { sqq. }])^{12}$.

Ou encore :

La sagesse et le courage sont autres chez un homme et chez une femme (un homme passerait pour lâche s'il n'était courageux que comme une femme courageuse, et une femme aurait des propos trop libres si elle n'avait que la réserve d'un homme de bien, puisque même la manière de tenir la maison est différente pour l'homme et pour la femme; le rôle de l'un étant d'acquérir, celui de l'autre, de conserver)

(ibid., iii.3 [1277 b 2 sqq.]).

Joli conflit d'idées : déjà au Ier siècle de notre ère Plutarque reprend ce débat dans son traité de la vertu des femmes, et prend le parti de Platon. Mais on retrouve ailleurs dans ses écrits moraux les traces du vocabulaire sexuel des stoïques, selon lequel l'épithète "femelle' équivaut à 'faible', 'corrompu', 'lâche', 'mou', 'inconstant', 'voué au plaisir', 'sujet aux passions' : épithètes à coloration aristotélicienne pour ne pas dire pythagorique ${ }^{13}$. Aussi existe-t-il au sein même de la philosophie idéaliste néoplatonique et stoïque, une incohérence tout aussi profonde que celle qu'on a décelée chez Galien : la femme est égale à l'homme (car l'un et l'autre sont constitués d'une âme et d'un corps, ayant en plus une volonté pour maîtriser celui-ci au profit de celle-là) : mais en même temps femelle n'est pas l'égal de mâle, car le premier terme incarne des oppositions hiérarchisées qui le rendent inférieur au second.

Que faire donc si son mécène s'appelle Elizabeth I ou la duchesse de Ferrare, et qu'on soit chargé d'élucider en bon humaniste la pensée politique des anciens? C'est le dilemme de trois érudits de la fin du XVI ${ }^{e}$ siècle dont j'esquisserai ici les réponses. Considérons d'abord John Case, philosophe néoaristotélicien, enseignant à Oxford, auteur en 1588 d'un commentaire sur la politique d'Aristote intitulé la Sphaera civitatis, qu'il place sous l'égide de son mécène Elizabeth. Le frontispice de son ouvrage la représente en sphère composée de cercles concentriques symbolisant les vertus de ce monarque : majestê, prudence, courage, religion, clémence, éloquence, abondance. D'après Case, l'affirmation du Stagirite selon laquelle le commandement c'est la perfection de la vertu masculine et la soumission celle de la vertu féminine ne pose pas de problème, car il ne s'ensuit pas de là que les femmes ne sont pas propres à commander, puisque les catégories homme/femme se chevauchent, comme on l'a vu, et qu'une femme fort chaude peut 
égaler le commun des hommes. Tout ce qu'on peut en déduire, c'est que les femmes en général ne sont pas propres à commander. De plus, il faut selon Case distinguer sphère domestique et sphère politique : il ne sied nullement à une épouse de commander à son mari (Saint Paul a dit son mot là-dessus aussi); mais une femme d'État peut commander aux hommes. Case ose même affirmer que la déclaration d'Aristote selon laquelle les femmes n'ont qu'un jugement (politique) imparfait (Bouגeutikòv akupov Politica, i. 5 [1260a11]) ne concerne que les épouses : or le texte d'Aristote n'admet pas cette interprétation. Rencherissant sur son hétérodoxie philosophique, Case prétend de plus que le tempérament n'a pas toujours de prise sur l'esprit, et que l'expérience de l'histoire nous enseigne qu'il y a eu jadis des femmes qui ont su jouer un rôle fort positif dans la vie politique. Parmi les exemples qu'il cite se trouve celui de Sparte : or Aristote nie explicitement que l'effet des femmes sur l'administration de cet État ait été bénéfique ${ }^{14}$. On retrouve donc chez Case une désarticulation du système d'Aristote au profit d'une apologie du règne des femmes sous une reinemécène : argument intéressé s'il en fut.

Le Tasse, lui, ne sombre pas si facilement dans l'incohérence philosophique. Dans son Discorso della virtù feminile et donnesca de 1582, il aspire au syncrétisme de la pensée platonique et de celle $\mathrm{d}^{\prime}$ Aristote. D'après le poète italien, chaque sexe possède une vertu dominante mais non exclusive : dans le cas de l'homme, c'est le courage; dans celui de la fermme, la chasteté. Un vice capital, antipode de la vertu dominante, s'attache aussi à chacun des deux sexes : lâcheté donc chez l'homme, impudicité chez la femme. Il s'ensuit que les femmes sont plus blâmables lorsqu'elles manquent à leur chasteté que quand elles s'avèrent lâches; et que, tout au contraire, l'on peut plus aisément pardonner aux hommes la débauche que la couardise. Cette vertu dominante n'est pas d'ailleurs la seule à déterminer les diverses responsabilités morales des deux sexes; éloquence, magnificence, libéralité (toute la gamme enfin des vertus dites héroïques) appartiennent plus aux mâles qu'aux femelles, à qui il siérait mieux de se montrer silencieuses, modestes, économes. Non que les femmes éloquentes, libérales et magnifiques soient vicieuses : mais ces qualités conviennent mieux aux hommes.

Tout cela est d'ordre éthique; que se passe-t-il si un devoir politique prévaut sur ces obligations morales? Une femme appelée à régner, qui est moralement tenue en tant que femme d'être silencieuse, modeste, économe, doit-elle en tant que monarque se 
montrer éloquente, magnifique, libérale? Pour répondre à cette question, Le Tasse distingue ordre moral et ordre politique selon leurs fins respectives : celle des vertus morales, c'est le bonheur de lindividu; celle des vertus politiques, c'est le bon fonctionnement de l'État. Les vertus morales se pratiquent sans contradiction chez les bourgeoises et les femmes de la petite noblesse: mais chez une personne royale, les devoirs de la vertu politique prévalent sur les devoirs moraux féminins : autrement dit, si cette femme erre plus du côté de l'impudicité que de celui de la lâcheté, cela est regrettable mais non repréhensible. C'est le cas, Le Tasse nous le rappelle, de Cléopâtre et de Sémiramis ${ }^{15}$. Tout comme John Case, le poète résout le problème conceptuel au sein de la pensée humaniste en le coupant de son contexte métaphysique; la raison politique et idéologique l'emporte sur la pureté de la doctrine. Dans d'autres textes (telle L'Aminta, pièce théâtraie publiée en 1573) Le Tasse se livre à des expériences de pensée inverses : il crée un monde antiaulique et pastoral où des réfugiés de la cour se libèrent des conventions de leur époque pour s'enquérir des vrais rapports humains tels que la Nature nous les dicte. Mais dans son discours de 1582 , il prône un dualisme social, peut-être pour défendre un mécène-femme qui aurait enfreint scandaleusement les lois de l'honneur féminin.

Si Case et Le Tasse ont l'image de leur mécène devant les yeux, on ne peut pas en dire autant d'Antonio Montecatini, patricien ferrarais et humaniste, qui s'impose la tâche de syncrétiser la pensée des anciens sur la politique. Son système, qu'il expose dans son traité In politica progymnasmata de 1587, part des principes moraux et politiques d'Aristote, qu'il cherche à concilier avec le concept de vertu générique chez Platon. Il prend le cas exemplaire du pater familias : celui-ci commande à sa femme, mais en tant que citoyen il obéit à l'état ou aux lois de la patrie ${ }^{16}$. Il possède donc deux types de vertus apparentes (une vertu pour commander, une pour obéir): Mais ces vertus ne se distinguent que superficiellement, car elles ne sont toutes les deux que des manifestations, fussent-elles contraires, de la même vertu générique. La différence n'est que d'application. Le père de famille bon citoyen n'est pas en contradiction profonde avec lui-même : il lui arrive de remplir des devoirs opposés en exerçant la même vertu générique selon un mode différent. Il en est de même pour les femmes : selon Montecatini, Platon a raison d'affirmer que les femmes ont la même vertu que les hommes; elles ont la même obligation d'être libérales, courageuses, justes; mais à leur propre 
façon, qui les contraint se montrer courageuses à force d'obéissance, libérales à force d'épargne, justes en se soumettant à la justice des hommes ${ }^{17}$. Montecatini préconise ici en dépit de l'affirmation néoplatonique de l'égalité des sexes en matière de vertu, un certain conformisme politique justifié par un fonctionnalisme qui n'est pas sans rappeler celui de Galien en physiologie, et qui s'accorde parfaitement à la répartition des rôles sexuels envisagés par Aristote. C'est grâce à une structure de pensée transformationnelle, qui fait que la veru commune aux deux se modifie en des manifestations contraires, que Montecatini arrive à concilier ces systèmes de pensée autrement irréconciliables. Il ne semble donc pas que les fondements métaphysiques de sa politique soient sacrifiés, comme chez Le Tasse et chez Case, sur lautel de l'opportunisme mécénique; mais noublions pas a quel point la pensté de Montecatini est profondément imprégnée de conservatisme et de conformisme. Pour trouver une pensée radicale qui menace l'idéologie de l'époque de Shakespeare, il faudrait chercher plus loin, chez des Ecrivains (et ils sont peu nombreax) qui renversent les systèmes philosophiques de leur époque et qui en rejettent l'autorité, tel Michel de Montaigne. Mais cela, c'est une autre histoire.

Comme je l'ai indiqué au début, cet exposé a êté fort incomplet; car en considérant isolément quelques éléments des discours médical et politique, j'ai omis de parler de l'appor de la théologie et du droit. Je n'ai donc pas de conclusion grandiose vous proposer; $\mathrm{j}$ 'ose simplement dire que l'étude de la différence sexuelle à la fin de la Renaissance nous révèle une structure conceptuelle fissurée, une pensée dont la cohérence est sans cesse menacée non seulement par la raison politique, ce cri strident de l'idéologie à l'état brut, mais aussi par des désarticulations intérieures. Tout cela transforme la différence sexuelle en opposition hiérarchisée à tous les niveaux: métaphysique, physiologique, psychologique, moral, politique. Les théoriciens néoplatoniques ou syncrétistes comme Le Tasse et Montecatini peuvent sembler égalitaires en revendiquant une même vertu pour les deux sexes, mais ils sont conservateurs en politique et ils n'abandonnent pas non plus l'usage d'un lexique stö̈que qui dévalorise la féminité. Les médecins galéniques, eux, croient célébrer la dignité du sexe féminin, mais ils continuent d'affirmer son infériorité psychologique. Les aristotéliciens tel Case essaient đ'adoucir les déclarations du mâtre au sujet des femmes, mais ils ne réussissent pas à ce libérer des structures profondes de la pensée d'Aristote, dont les antithèses fondatrices --. âme corps; forme/matière; acte/puissance; 
possession/ privation - viennent toutes à l'appui de la supériorité mâle. Tous ces textes équivoques nous portent enfin à y lire en filigrane les oppositions pythagoriques - mâle/fini/impair/un/ droite/carré/immobile/ etc. - par lesquelles j'ai commencé cet exposé, et qui peut-être encore aujourd'hui influent sur nous plus que nous ne serions prêts à l'admettre.

Ian MACLEAN

The Queen's College, Oxford

\section{NOTES}

1. Voir The Renaissance notion of woman: a study in the fortunes of scholasticism and medical science in European intellectual life, Cambridge, 1980.

2. 'Small Latine and lesse Greeke', To the memory of my beloved, the author, Mr William Shakespeare, v. 31, Works ed. C.H. Herford, Percy \& Evelyn Simpson, Oxford, 1947, viii.391.

3. Voir F. Edward Cranz, A bibliography of Aristotle editions 1501-1600, revisée par Charles B. Schmitt, Baden-Baden, 1984 (Bibliotheca bibliographica aureliana $x \times x v i i^{\circ}$ ).

4. Occulta naturae miracula (1559), Antwerp, 1574, I. 9, pp. 3945; Maclean, Renaissance notion of woman, pp. 28 sqq., p. 106.

5. Ibid., p. 8; pp. 28 sqq. C'est là l'élaboration d'une idée de Platon (Timaeus, 50c) : voir aussi Aristote, Physica, i.9 (192 a 22): Maclean, Renaissance notion of woman, pp. 40 sqq.

6. De generatione animalium ii.3 (383 a 27); iv.6 (775 a 16 sqq.).

7. Les epithetes (1573), Lyons, 1593 , ff. $173^{\mathrm{V}}-4^{\mathrm{T}}$.

8. Catégoriae, X (11 b 16 sqq.).

9. De usu paratium corporis, XIV; De semine, II.

10. Maclean, Renaissance notion of woman, pp. 30 sqq.

11. Les passions de l'âme, art. 50, dans $C$ Euvres et lettres, éd. André Bridoux, Paris 1953, pp. 721-2.

12. Aristote cite ici Sophocle, Ajax, 293: voir aussi Politica, v. 9 (1310 a 12 sqq.), Ethica ad Nicomacheum, v. 2 (1130 b 25 sqq.). p. 53.

13. Mulierum virtutes; Maclean, Renaissance notion of woman,

14. Ibid., pp. $60-1$; Politica, ii.9 (1269b12 sqq.).

15. Maclean, Renaissance notion of woman, pp. 61-3.

16. Voir Politica, i.5 (1259 a 38 sqq.).

17. In politica progymnasmata, Ferrare, 1587, pp. 49 sqq. 